\title{
Investigation of Photochemical Reactions of some Metal Carbonyls with N'-(2-Hydroxy-6- Methylbenzylidene)Methanesulfonohydrazide and 5-Methyl- 2-Hydroxyacetophenonemethanesulfonylhydrazone
}

\author{
Sema Sert ${ }^{1}$ \\ ${ }^{1}$ Department of Chemistry, Faculty of Science, Ege University, Izmir, Turkey \\ *Corresponding author: E-mail: semasertkimya@hotmail.com
}

Received: 05-08-2019

\begin{abstract}
Eight new complexes, $\left[\mathrm{M}(\mathrm{CO})_{5}(5 \mathrm{msalmsh})\right][\mathrm{M}=\mathrm{Cr} ;(1 \mathrm{a}), \mathrm{Mo} ;(2 \mathrm{a}) ; \mathrm{W}(3 \mathrm{a})],\left[\operatorname{Re}(\mathrm{CO})_{4} \mathrm{Br}(5 \mathrm{msalmsh})\right](4 \mathrm{a}),\left[\mathrm{M}(\mathrm{CO})_{5}(5 \mathrm{ma}-\right.$ fmsh)] [M=Cr;(1b), Mo;(2b); W(3b)], $\left[\operatorname{Re}(\mathrm{CO})_{4} \operatorname{Br}(5 \mathrm{mafmsh})\right](4 \mathrm{~b})$, have been synthesized by the photochemical reaction of the metal carbonyls $\left[\mathrm{M}(\mathrm{CO})_{6}\right](\mathrm{M}=\mathrm{Cr}, \mathrm{Mo}, \mathrm{W})$ and $\left[\mathrm{Re}(\mathrm{CO})_{5} \mathrm{Br}\right]$ with $\mathrm{N}^{\prime}$-(2-Hydroxy-6-Methylbenzylidene) Methanesulfonohydrazide (5msalmsh) and 5-methyl-2-hydroxyacetophenonemethanesulfonylhydrazone (5mafmsh). The complexes have been characterized by elemental analysis, LC-MS, FT-IR, ${ }^{1} \mathrm{H}$ NMR spectroscopy. Spectroscopic studies show that $5 \mathrm{msalmsh}$ and $5 \mathrm{mafmsh}$ behave monodentate ligand and coordinate via an imine $\mathrm{N}$ donor atom to the central metal atom in (1a)-(4a) and (1b)-(4b).
\end{abstract}

Keywords: Hydrazones; monodentate ligands; metal carbonyls; photochemical reactions.

\section{Introduction}

Sulfonylhyrazones and sulfonamides have been shown to be active in several pharmacological tests, demostrating antibacterial, antitumor, diuretic, antiviral, and antinociceptive activity. ${ }^{1-5}$ These compounds have gained importance in bioinorganic and metal based drug chemistry because of their lower cost, lower toxicity and most activity against bacterial diseases. ${ }^{6,7}$ Moreover, they are used in agriculture field as well as insecticides and herbicides. They are less toxic as compared to other drugs and are scalable., ${ }^{8,9}$

In chemical synthesis, photochemical reactions are usually cleaner and more efficient than other types of reactions because the key reagent is light of particular energy. In fine chemical synhhesis and pharmaceutical productions, photochemical reaction steps offer shorter routes for many synthetic schemes, e.g. synthesis of vitamin D. In addition, photocleavage has also become one of the more effective methods for removing protective groups. The use of photochemistry is limited by concerns about scalability, efficiency, and safe operations of the processes. ${ }^{10}$

Metal carbonyl complexes are among the most photoreactive transition metal complexes known. ${ }^{11}$ The commonly known carbonyls of $\mathrm{Cr}(0), \operatorname{Mo}(0)$, and $\mathrm{W}(0)$ are six-coordinate octahedral complexes, $\mathrm{M}(\mathrm{CO})_{6}$. Other stable complexes containing only the central metal and $\mathrm{CO}$ include the dimers $\mathrm{M}_{2}(\mathrm{CO})_{10}{ }^{2-}$ having a single $\mathrm{M}-\mathrm{M}$ bond. Numerous compounds of the $\mathrm{M}(\mathrm{CO})_{\mathrm{n}}(\mathrm{L})_{6}{ }_{\mathrm{n}} \mathrm{n}$ variety have been prepared, many photochemically. ${ }^{12}$

In this work, eight new complexes $\left[\mathrm{M}(\mathrm{CO})_{5}(5 \mathrm{~m}\right.$ salmsh)] [M=Cr;(1a), Mo;(2a); W(3a)], $\left[\operatorname{Re}(\mathrm{CO}){ }_{4} \mathrm{Br}(5 \mathrm{~m}-\right.$ salmsh)] (4a), [M(CO) 5 (5mafmsh)] [M=Cr;(1b), Mo;(2b); $\mathrm{W}(3 \mathrm{~b})],\left[\operatorname{Re}(\mathrm{CO})_{4} \mathrm{Br}(5 \mathrm{mafmsh})\right](4 \mathrm{~b})$ as shown in Scheme 1 were synthesized by the photochemical reactions of<smiles>[R]C(=NNS(C)(=O)=O)c1cc(C)ccc1O</smiles>

5 msalmsh; $\mathrm{R}=\mathrm{H}$ 5mafmsh; $\mathrm{R}=\mathrm{CH}_{3}$

Figure 1. The structure of ligands. 
$\left[\mathrm{M}(\mathrm{CO})_{6}\right](\mathrm{Cr}, \mathrm{Mo}, \mathrm{W})$ with $5 \mathrm{msalmsh}$ and $5 \mathrm{mafmsh}$ (shown in Figure 1) and characterized by elemental analysis, MS, IR and ${ }^{1} \mathrm{H}$ NMR spectroscopy. According to all the spectroscopic data, $5 \mathrm{msalmsh}$ and $5 \mathrm{mafmsh}$ are monodentate and coordinated via the imine nitrogen donor atom.

\section{Experimental}

\section{1. Physical Measurements}

Reactions were carried out under dry nitrogen using Schlenk techniques. All solvents were dried and degassed prior to use. Elemental analyses were performed according to standard micro analytical procedures (TÜBİTAK Laboratories, Ankara). The infrared spectra of the compounds as $\mathrm{KBr}$ disks were recorded in the range of $4000-400 \mathrm{~cm}^{-1}$ with a Mattson 1000 FT spectrometer. ${ }^{1} \mathrm{H}$ NMR spectra of dimethylsulfoxide- $\mathrm{d}_{6}\left(\right.$ DMSO- $\left.\mathrm{d}_{6}\right)$ solutions of the compounds were recorded on a $400 \mathrm{MHz}$ digital FT-NMR at TÜBİTAK. Electron impact mass spectra were recorded on a Micromass VG Platform-II LC-MS at TÜBİTAK. Photochemical reactions were carried out in an immersion-well apparatus by using a medium pressure $400 \mathrm{~W}$ Mercury lamp .All solvents and silica gel were purchased from Merck. $\mathrm{M}(\mathrm{CO})_{6}(\mathrm{M}=\mathrm{Cr}, \mathrm{Mo}, \mathrm{W})$ and $\operatorname{Re}(\mathrm{CO})_{5} \mathrm{Br}$ were purchased from Aldrich. These reagents were used as supplied. $5 \mathrm{msalmsh}$ and $5 \mathrm{mafmsh}$ were prepared by the literature method. ${ }^{13}$

\section{2. Synthesis}

Complexes (1a)-(4a) and (1b)-(4b) were prepared by photochemical reactions of metal carbonyls $\mathrm{M}(\mathrm{CO})_{6}$ $(\mathrm{M}=\mathrm{Cr}, \mathrm{Mo}, \mathrm{W})$ and $\operatorname{Re}(\mathrm{CO})_{5} \mathrm{Br}$ with $5 \mathrm{msalmsh}$ and $5 \mathrm{mafmsh}$, and were obtained in $50-70 \%$ yields. The methods employed for the preparation of the complexes are very similar, so that the preparation of $\left[\mathrm{Cr}(\mathrm{CO})_{5}(5 \mathrm{~m}\right.$ salmsh)] (1a) is given in detail as a representative example.

\section{2. 1. $\left[\mathrm{Cr}(\mathrm{CO})_{5}(5 \mathrm{msalmsh})\right],(1 \mathrm{a})$.}

$\mathrm{Cr}(\mathrm{CO})_{6}(0.44 \mathrm{~g}, 2 \mathrm{mmol})$ and $5 \mathrm{msalmsh}(0.44 \mathrm{~g}, 2$ $\mathrm{mmol})$ were dissolved in THF $(80-100 \mathrm{~mL})$. The solution was irradiated for $2 \mathrm{~h}$ at room temperature. During irradiation, the reaction mixture changed from colorless to dark yellow. After dissolving in dichloromethane $\left(10 \mathrm{~cm}^{3}\right)$, petroleum ether $\left(50 \mathrm{~cm}^{3}\right)$ was added, resulting in the precipitation of a dark yellow solid which was washed with petroleum ether and dried under vacuum. Yield (60\%). Found (\%): C, 38.8; H, 2.5; N, 6.4; S, 7.2. Calcd. for $\mathrm{CrC}_{14} \mathrm{H}_{12} \mathrm{~N}_{2} \mathrm{SO}_{8}$ (\%): C, 40.0; H, 2.9; N, 6.7; S, 7.6. IR (v, $\mathrm{KBr}): 2067$ (m, CO), 1978 (m, CO), 1950 (s, CO), 1928 (s, CO), 1873 (s, CO), 3158 (s, N-H), 1602(s, C=N), 1315 (s, $\mathrm{C}-\mathrm{O}), 1274\left(\mathrm{~s},\left(\mathrm{SO}_{2}\right)_{\mathrm{as}}\right), 1152\left(\mathrm{~s},\left(\mathrm{SO}_{2}\right)_{\mathrm{sym}}\right) \mathrm{cm}^{-1} .{ }^{1} \mathrm{H} \mathrm{NMR}$ $\left(\delta, \mathrm{DMSO}_{-} \mathrm{d}_{6}\right): 2.10\left(\mathrm{~s}, 3 \mathrm{H}, \mathrm{CH}_{3}-\mathrm{C}_{6} \mathrm{H}_{5}\right), 2.94\left(\mathrm{~s}, 3 \mathrm{H}, \mathrm{CH}_{3^{-}}\right.$ $\left.\mathrm{SO}_{2}\right), 6.56-7.20\left(\mathrm{~m}, \mathrm{H},(\mathrm{CH})_{\mathrm{Ar}}\right), 7.95(\mathrm{~s}, 1 \mathrm{H}, \mathrm{HC}=\mathrm{N}-), 9.98$ (s, H, NH), $11.87(\mathrm{~s}, 1 \mathrm{H}, \mathrm{OH}) \cdot \mathrm{MS}(\mathrm{LC}, 70 \mathrm{eV}): \mathrm{m} / \mathrm{z}(\%)=$ 405 (25) $\left[\mathrm{M}^{+}-(\mathrm{Me})\right], 377$ (15) $\left[\mathrm{M}^{+}-(\mathrm{Me}+\mathrm{CO}], 349\right.$ (30) $\left[\mathrm{M}^{+}-(\mathrm{Me}+2 \mathrm{CO}], 321 \quad(25) \quad\left[\mathrm{M}^{+}-(\mathrm{Me}+3 \mathrm{CO}], 293\right.\right.$ (15) $\left[\mathrm{M}^{+}-(\mathrm{Me}+4 \mathrm{CO}], 265(10)\left[\mathrm{M}^{+}-(\mathrm{Me}+5 \mathrm{CO}]\right.\right.$.

\section{2. 2. $\left[\mathrm{Mo}(\mathrm{CO})_{5}(5 \mathrm{msalmsh})\right],(2 \mathrm{a})$.}

Yield (64\%). Found (\%): C, 35.6; H, 2.5; N, 5.6; S, 6.5. Calcd. for $\mathrm{MoC}_{14} \mathrm{H}_{12} \mathrm{~N}_{2} \mathrm{SO}_{8}$ (\%): C, 36.1; $\mathrm{H}, 2.6 ; \mathrm{N}, 6.0 ; \mathrm{S}$, 6.9. IR (v, KBr): 2066 (m, CO), 1990 (m, CO), 1950 (s, CO), 1925 (s, CO), 1871 (s, CO), 3160 (s, N-H), 1602(s, $\mathrm{C}=\mathrm{N}), 1315(\mathrm{~s}, \mathrm{C}-\mathrm{O}), 1274\left(\mathrm{~s},\left(\mathrm{SO}_{2}\right)_{\mathrm{as}}\right), 1152\left(\mathrm{~s},\left(\mathrm{SO}_{2}\right)_{\mathrm{sym}}\right)$ $\mathrm{cm}^{-1} .{ }^{1} \mathrm{H}$ NMR $\left(\delta\right.$, DMSO-d $\left.\mathrm{d}_{6}\right): 2.08\left(\mathrm{~s}, 3 \mathrm{H}, \mathrm{CH}_{3}-\mathrm{C}_{6} \mathrm{H}_{5}\right)$, $2.96\left(\mathrm{~s}, 3 \mathrm{H}, \mathrm{CH}_{3}-\mathrm{SO}_{2}\right), 6.58-7.14\left(\mathrm{~m}, \mathrm{H},(\mathrm{CH})_{\mathrm{Ar}}\right), 7.93$ $(\mathrm{s}, 1 \mathrm{H}, \mathrm{HC}=\mathrm{N}-), 9.96(\mathrm{~s}, \mathrm{H}, \mathrm{NH}), 11.88(\mathrm{~s}, 1 \mathrm{H}, \mathrm{OH}) . \mathrm{MS}$ $(\mathrm{LC}, 70 \mathrm{eV}): \mathrm{m} / \mathrm{z}(\%)=436(15)\left[\mathrm{M}^{+}-(\mathrm{CO})\right], 408(25)\left[\mathrm{M}^{+}-\right.$ (2CO)], $380(25)\left[\mathrm{M}^{+}-(3 \mathrm{CO})\right], 352(15)\left[\mathrm{M}^{+}-(4 \mathrm{CO})\right], 324$ (10) $\left[\mathrm{M}^{+}-(5 \mathrm{CO})\right]$.

\section{2. 3. $\left[\mathrm{W}(\mathrm{CO})_{5}(5 \mathrm{msalmsh})\right],(3 \mathrm{a})$.}

Yield (62\%). Found (\%): C, 29.4; H, 2.0; N, 5.0; S, 5.2. Calcd. for $\mathrm{WC}_{14} \mathrm{H}_{12} \mathrm{~N}_{2} \mathrm{SO}_{8}$ (\%): C, 30.4; $\mathrm{H}, 2.2 ; \mathrm{N}, 5.1$; S,5.8. IR (v, KBr): 2065 (m, CO), 1991 (m, CO), 1955 (s, $\mathrm{CO}), 1922$ (s, CO), 1872 (s, CO), 3158(s, N-H), 1602 (s, $\mathrm{C}=\mathrm{N}), 1315(\mathrm{~s}, \mathrm{C}-\mathrm{O}), 1274\left(\mathrm{~s},\left(\mathrm{SO}_{2}\right)_{\mathrm{as}}\right), 1152\left(\mathrm{~s},\left(\mathrm{SO}_{2}\right)_{\mathrm{sym}}\right)$ $\mathrm{cm}^{-1} .{ }^{1} \mathrm{H}$ NMR $\left(\delta\right.$,DMSO-d $\left.\mathrm{d}_{6}\right): 2.14\left(\mathrm{~s}, 3 \mathrm{H}, \mathrm{CH}_{3}-\mathrm{C}_{6} \mathrm{H}_{5}\right)$, $2.92\left(\mathrm{~s}, 3 \mathrm{H}, \mathrm{CH}_{3}-\mathrm{SO}_{2}\right), 6.58-7.14\left(\mathrm{~m}, \mathrm{H},(\mathrm{CH})_{\mathrm{Ar}}\right), 7.94(\mathrm{~s}$, $1 \mathrm{H}, \mathrm{HC}=\mathrm{N}-), 9.94(\mathrm{~s}, \mathrm{H}, \mathrm{NH}), 11.85(\mathrm{~s}, 1 \mathrm{H}, \mathrm{OH}) . \mathrm{MS}$ $(\mathrm{LC}, 70 \mathrm{eV}): \mathrm{m} / \mathrm{z}(\%)=537(15)\left[\mathrm{M}^{+}-(\mathrm{Me})\right], 509(10)$ $\left[\mathrm{M}^{+}-(\mathrm{Me}+\mathrm{CO}], 481 \quad(10) \quad\left[\mathrm{M}^{+}-(\mathrm{Me}+2 \mathrm{CO}], 453\right.\right.$ (20) $\left[\mathrm{M}^{+}-(\mathrm{Me}+3 \mathrm{CO}], 397(15)\left[\mathrm{M}^{+}-(\mathrm{Me}+5 \mathrm{CO}]\right.\right.$.

\section{2. 4. $\left[\operatorname{Re}(\mathrm{CO})_{4} \operatorname{Br}(5 \mathrm{msalmsh}],(4 \mathrm{a})\right.$.}

Yield (58\%). Found(\%): C, 25.4; H, 21.8; N, 4.2; S, 5.1. Calcd. for $\mathrm{ReC}_{13} \mathrm{H}_{12} \mathrm{~N}_{2} \mathrm{SO}_{7} \mathrm{Br}(\%)$ : C, 25.7; H, 2.0; N, 4.6; S, 5.3. IR (v, KBr): 2112 (w, CO), 2016 (m, CO), 1965 (m, CO), 1932 (s, CO), 3158 (s, N-H), 1602(s, C=N), 1315 (s, C-O), $1274\left(\mathrm{~s},\left(\mathrm{SO}_{2}\right)_{\mathrm{as}}\right), 1152\left(\mathrm{~s},\left(\mathrm{SO}_{2}\right)_{\mathrm{sym}}\right) \mathrm{cm}^{-1} \cdot{ }^{1} \mathrm{H}$ $\operatorname{NMR}\left(\delta\right.$, DMSO-d $\left.\mathrm{d}_{6}\right): 2.12\left(\mathrm{~s}, 3 \mathrm{H}, \mathrm{CH}_{3}-\mathrm{C}_{6} \mathrm{H}_{5}\right), 2.90(\mathrm{~s}, 3 \mathrm{H}$, $\left.\mathrm{CH}_{3}-\mathrm{SO}_{2}\right), 6.56-7.12\left(\mathrm{~m}, \mathrm{H},(\mathrm{CH})_{\mathrm{Ar}}\right), 7.92(\mathrm{~s}, 1 \mathrm{H}, \mathrm{HC}=\mathrm{N}-$ ), $9.96(\mathrm{~s}, \mathrm{H}, \mathrm{NH}), 11.86(\mathrm{~s}, 1 \mathrm{H}, \mathrm{OH}) . \mathrm{MS}(\mathrm{LC}, 70 \mathrm{eV}): \mathrm{m} / \mathrm{z}$ $(\%)=578(15)\left[\mathrm{M}^{+}-(\mathrm{CO})\right], 550(15)\left[\mathrm{M}^{+}-(2 \mathrm{CO})\right], 494(20)$ $\left[\mathrm{M}^{+}-(4 \mathrm{CO})\right]$.

\section{2. 5. $\left[\mathrm{Cr}(\mathrm{CO})_{5}(5 \mathrm{mafmsh})\right],(1 \mathrm{~b})$.}

Yield (62\%). Found(\%): C, 41.2; H, 2.9; N, 6.0; S, 7.0. Calcd. for $\mathrm{CrC}_{15} \mathrm{H}_{14} \mathrm{~N}_{2} \mathrm{SO}_{8}$ (\%): C, 41.5; H, 3.2; N, 6.4; S, 7.4. IR (v, KBr): 2071 (m, CO), 1970 (m, CO), 1946 (s, CO), 1936 (s, CO), 1872 (m, CO), 3204 (s, N-H), 1608 (m, C=N), 1323 (s, C-O), $1254\left(\mathrm{~s},\left(\mathrm{SO}_{2}\right)_{\mathrm{as}}\right), 1150\left(\mathrm{~s},\left(\mathrm{SO}_{2}\right)_{\mathrm{sym}}\right) \mathrm{cm}^{-1} \cdot{ }^{1} \mathrm{H}$ $\operatorname{NMR}\left(\delta\right.$, DMSO-d $\left.{ }_{6}\right): 2.20\left(\mathrm{~s}, 3 \mathrm{H}, \mathrm{CH}_{3}-\mathrm{C}_{6} \mathrm{H}_{5}\right), 2.98(\mathrm{~s}, 3 \mathrm{H}$, $\left.\mathrm{CH}_{3}-\mathrm{SO}_{2}\right), 6.76-7.28\left(\mathrm{~m}, \mathrm{H},(\mathrm{CH})_{\mathrm{Ar}}\right), 2.20\left(\mathrm{~s}, 3 \mathrm{H}, \mathrm{CH}_{3^{-}}\right.$ $\mathrm{C}=\mathrm{N}-), 10.86(\mathrm{~s}, \mathrm{H}, \mathrm{NH}), 11.50(\mathrm{~s}, 1 \mathrm{H}, \mathrm{OH}) . \mathrm{MS}(\mathrm{LC}, 70 \mathrm{eV})$ : 
$\mathrm{m} / \mathrm{z}(\%)=433(10)\left[\mathrm{M}^{+}-(3 \mathrm{H})\right], 418(15)\left[\mathrm{M}^{+}-(3 \mathrm{H}+\mathrm{Me})\right]$, 390 (15) $\left[\mathrm{M}^{+}-(3 \mathrm{H}+\mathrm{Me}+\mathrm{CO})\right], 362(20)\left[\mathrm{M}^{+}-(3 \mathrm{H}+\mathrm{Me}+-\right.$ $2 \mathrm{CO})], \quad 334$ (15) $\left[\mathrm{M}^{+}-(3 \mathrm{H}+\mathrm{Me}+3 \mathrm{CO})\right], \quad 306 \quad$ (10) $\left[\mathrm{M}^{+}-(3 \mathrm{H}+\mathrm{Me}+4 \mathrm{CO})\right], 278(15)\left[\mathrm{M}^{+}-(3 \mathrm{H}+\mathrm{Me}+5 \mathrm{CO})\right]$.

\section{2. 6. $\left[\mathrm{Mo}(\mathrm{CO})_{5}(5 \mathrm{mafmsh})\right],(2 \mathrm{~b})$.}

Yield (66\%). Found(\%): C, 36.8; H, 2.7; N, 5.4; S, 6.2. Calcd. for $\mathrm{MoC}_{15} \mathrm{H}_{14} \mathrm{~N}_{2} \mathrm{SO}_{8}$ (\%): C, 37.6; H, 2.9; N, 5.8; S, 6.7. IR (v, KBr): 2070 (m, CO), 1971 (m, CO), 1944 (s, CO), 1925 (s, CO), 1872 (m, CO), 3203 (s, N-H), 1608 (m, $\mathrm{C}=\mathrm{N}), 1323$ (s, C-O), $1252\left(\mathrm{~s},\left(\mathrm{SO}_{2}\right)_{\mathrm{as}}\right), 1152\left(\mathrm{~s},\left(\mathrm{SO}_{2}\right)_{\mathrm{sym}}\right)$ $\mathrm{cm}^{-1} .{ }^{1} \mathrm{H}$ NMR $\left(\delta\right.$, DMSO-d $\left.\mathrm{d}_{6}\right): 2.18\left(\mathrm{~s}, 3 \mathrm{H}, \mathrm{CH}_{3}-\mathrm{C}_{6} \mathrm{H}_{5}\right)$, $3.02\left(\mathrm{~s}, 3 \mathrm{H}, \mathrm{CH}_{3}-\mathrm{SO}_{2}\right), 6.77-7.20\left(\mathrm{~m}, \mathrm{H},(\mathrm{CH})_{\mathrm{Ar}}\right), 2.24(\mathrm{~s}$, $\left.3 \mathrm{H}, \mathrm{CH}_{3}-\mathrm{C}=\mathrm{N}-\right), 10.87$ (s, H, NH), 11.58 (s, 1H, OH). MS $(\mathrm{LC}, 70 \mathrm{eV}): \mathrm{m} / \mathrm{z}(\%)=420(20)\left[\mathrm{M}^{+}-(2 \mathrm{Me}+\mathrm{CO}], 492(15)\right.$ $\left[\mathrm{M}^{+}-(2 \mathrm{Me}+2 \mathrm{CO}], 364(15)\left[\mathrm{M}^{+}-(2 \mathrm{Me}+3 \mathrm{CO}], 336\right.\right.$ (25) $\left[\mathrm{M}^{+}-(2 \mathrm{Me}+4 \mathrm{CO}], 308(20)\left[\mathrm{M}^{+}-(2 \mathrm{Me}+5 \mathrm{CO}]\right.\right.$.

\section{2. 7. $\left[\mathrm{W}(\mathrm{CO})_{5}(5 \mathrm{mafmsh})\right],(3 \mathrm{~b})$.}

Yield (64\%). Found(\%): C, 31.2; H, 2.3; N, 4.4; S, 5.2. Calcd. for $\mathrm{WC}_{15} \mathrm{H}_{14} \mathrm{~N}_{2} \mathrm{SO}_{8}(\%)$ : C, 31.8; H, 2.5; N, 4.9; S, 5.6. IR (v, KBr): 2064 (m, CO), 1972 (m, CO), 1932 (s, CO), 1921 (s, CO), 1875 (m, CO), 3204 (s, N-H), 1609 (m, C=N), 1324 (s, C-O), $1253\left(\mathrm{~s},\left(\mathrm{SO}_{2}\right)_{\mathrm{as}}\right), 1150\left(\mathrm{~s},\left(\mathrm{SO}_{2}\right)_{\mathrm{sym}}\right) \mathrm{cm}^{-1} \cdot{ }^{1} \mathrm{H}$ $\operatorname{NMR}(\delta$, DMSO-d $): 2.22\left(\mathrm{~s}, 3 \mathrm{H}, \mathrm{CH}_{3}-\mathrm{C}_{6} \mathrm{H}_{5}\right), 3.01(\mathrm{~s}, 3 \mathrm{H}$,
$\left.\mathrm{CH}_{3}-\mathrm{SO}_{2}\right), 6.75-7.28\left(\mathrm{~m}, \mathrm{H},(\mathrm{CH})_{\mathrm{Ar}}\right), 2.22\left(\mathrm{~s}, 3 \mathrm{H}, \mathrm{CH}_{3^{-}}\right.$ $\mathrm{C}=\mathrm{N}-), 10.88$ (s, H, NH), $11.56(\mathrm{~s}, 1 \mathrm{H}, \mathrm{OH})$. MS (LC,70 eV): $\mathrm{m} / \mathrm{z}(\%)=551(10)\left[\mathrm{M}^{+}-(\mathrm{Me})\right], 523(10)\left[\mathrm{M}^{+}-(\mathrm{Me}+\mathrm{CO}]\right.$, 495 (15) $\left[\mathrm{M}^{+}-(\mathrm{Me}+2 \mathrm{CO}], 467\right.$ (20) $\left[\mathrm{M}^{+}-(\mathrm{Me}+3 \mathrm{CO}], 439\right.$ (25) $\left[\mathrm{M}^{+}-(\mathrm{Me}+4 \mathrm{CO}], 411(10)\left[\mathrm{M}^{+}-(\mathrm{Me}+5 \mathrm{CO}]\right.\right.$.

\section{2. 8. $\left[\operatorname{Re}(\mathrm{CO})_{4} \operatorname{Br}(5 \mathrm{mafmsh}],(4 \mathrm{~b})\right.$.}

Yield (60\%). Found(\%): C, 26.8; H, 2.0; N, 4.0; S, 5.0. Calcd. for $\mathrm{ReC}_{14} \mathrm{H}_{14} \mathrm{~N}_{2} \mathrm{SO}_{7} \mathrm{Br}(\%)$ : C, 27.1; H, 2.2; N, 4.5; S, 5.2. IR (v, KBr): 2113 (w, CO), 2022 (m, CO), 1920 (m, CO), 1908 (s, CO), 3204 (s, N-H), 1610(m, C=N), 1324 (s, $\mathrm{C}-\mathrm{O}), 1253\left(\mathrm{~s},\left(\mathrm{SO}_{2}\right)_{\mathrm{as}}\right), 1152\left(\mathrm{~s},\left(\mathrm{SO}_{2}\right)_{\mathrm{sym}}\right) \mathrm{cm}^{-1} \cdot{ }^{1} \mathrm{H} \mathrm{NMR}$ $\left(\delta, \mathrm{DMSO}_{6}\right): 2.16\left(\mathrm{~s}, 3 \mathrm{H}, \mathrm{CH}_{3}-\mathrm{C}_{6} \mathrm{H}_{5}\right), 2.96\left(\mathrm{~s}, 3 \mathrm{H}, \mathrm{CH}_{3}-\right.$ $\left.\mathrm{SO}_{2}\right), 6.76-7.25\left(\mathrm{~m}, \mathrm{H},(\mathrm{CH})_{\mathrm{Ar}}\right), 2.28\left(\mathrm{~s}, 1 \mathrm{H}, \mathrm{CH}_{3}-\mathrm{C}=\mathrm{N}-\right)$, 10.84 (s, H, NH), 11.58 (s, 1H, OH). MS (LC,70 eV): m/z $(\%)=617(20)\left[\mathrm{M}^{+}-(3 \mathrm{H})\right], 602(10)\left[\mathrm{M}^{+}-(3 \mathrm{H}+\mathrm{Me})\right], 546$ (25) $\left[\mathrm{M}^{+}-(3 \mathrm{H}+\mathrm{Me}+2 \mathrm{CO})\right], \quad 518$ (25) $\left[\mathrm{M}^{+}-(3 \mathrm{H}+\mathrm{Me}+-\right.$ $3 \mathrm{CO})], 490$ (15) $\left[\mathrm{M}^{+}-(3 \mathrm{H}+\mathrm{Me}+4 \mathrm{CO})\right]$.

\section{Results and Discussion}

Complexes (1a-4a) and (1b-4b) were prepared by a photochemical reaction as shown in Scheme 1. The photogeneration of $\mathrm{M}(\mathrm{CO})_{5}$ from $\mathrm{M}(\mathrm{CO})_{6}(\mathrm{M}=\mathrm{Cr}, \mathrm{Mo}, \mathrm{W})$ has been extensively studied. The 16-electron $\mathrm{M}(\mathrm{CO})_{5}$ frag-<smiles>[R]/C(=N/NS(C)(=O)=O)c1cc(C)ccc1O</smiles>

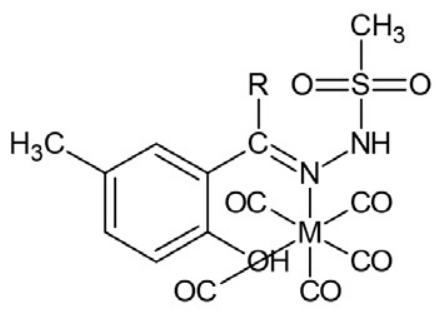<smiles>[Mg]</smiles>

5msalmsh, $\mathrm{R}=\mathrm{H}$

5 mafmsh, $\mathrm{R}=\mathrm{CH}_{3}$

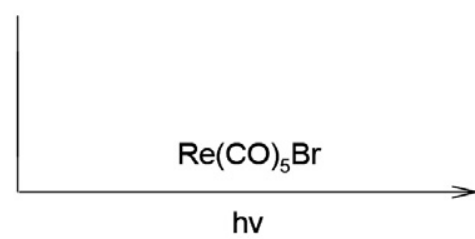

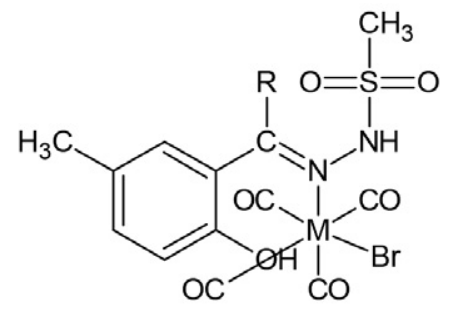

(4)

Scheme 1. The photochemical reaction of $\mathrm{M}(\mathrm{CO})_{6}(\mathrm{M}=\mathrm{Cr}, \mathrm{Mo}, \mathrm{W})$ and $\mathrm{Re}(\mathrm{CO})_{5} \mathrm{Br}$ with the $5 \mathrm{msalmsh}$ and $5 \mathrm{mafmsh}$ ligands. 
ments react quickly with any available donor atom to form a $\mathrm{M}(\mathrm{CO})_{5} \mathrm{~L}$ species. If $\mathrm{L}$ is a bidentate ligand, $\mathrm{M}(\mathrm{CO})_{4} \mathrm{~L}$ chelate or bridging $\mathrm{M}_{2}(\mathrm{CO})_{10}(\mu-\mathrm{L})$ compounds may occur. ${ }^{14-16}$ In this study, photochemical reactions of $\mathrm{M}(\mathrm{CO})_{6}$ $(\mathrm{M}=\mathrm{Cr}, \mathrm{Mo}, \mathrm{W})$, and $\mathrm{Re}(\mathrm{CO})_{5} \mathrm{Br}$ with $5 \mathrm{msalmsh}$ and $5 \mathrm{mafmsh}$ ligands proceed in this expected manner and give a series of complexes ( $1 \mathrm{a}-4 \mathrm{a})$ and $(\mathrm{b}-4 \mathrm{~b})$.

The strong $\mathrm{C}=\mathrm{N}$ stretching vibration, found at 1618 $\mathrm{cm}^{-1}$ in $5 \mathrm{msalmsh}$, shifts to lower wavenumber in (1a-4a) and the strong $\mathrm{C}=\mathrm{N}$ stretching vibration, found at 1628 $\mathrm{cm}^{-1}$ in $5 \mathrm{mafmsh}$, shifts to lower wavenumber in (1b-4b), showing that $5 \mathrm{msalmsh}$ and $5 \mathrm{mafmsh}$ ligands coordinate to the metal via the imine donor atom. ${ }^{16}$ These shifts have been explained as a weakening of the $\mathrm{CN}$ bond resulting from the loss of electron density from the nitrogen to the metal atom. ${ }^{17}$ No shifting upon complex formation was observed for the $v_{\text {as }}\left(\mathrm{SO}_{2}\right), \mathrm{M}_{\text {sym }}\left(\mathrm{SO}_{2}\right), v(\mathrm{NH})$ and $v(\mathrm{CO})$ stretching vibrations indicating that $\mathrm{SO}_{2}, \mathrm{NH}$ and $\mathrm{CO}$ groups were not coordinated to metal atom in $(1 \mathrm{a}-4 \mathrm{~b})$ and (1b-4b). The $\mathrm{OH}$ stretching vibration was not observed in both free ligand as well as in $(1 \mathrm{a}-4 \mathrm{a})$ and $(1 \mathrm{~b}-4 \mathrm{~b})$ because of hydrogen bonding with the imine nitrogen atom. ${ }^{18}$

According to number of carbonyl bands, provides important clues to the environment of the metal centers. ${ }^{19}$ Five carbonyl stretching bands in ( $1 \mathrm{a}-3 \mathrm{a})$ and $(1 \mathrm{~b}-3 \mathrm{~b})$ are attributed to local $\mathrm{C}_{4 \mathrm{v}}$ symmetry $\mathrm{M}(\mathrm{CO})_{5}$. Similarly four $\mathrm{CO}$ stretching absorptions in $(4 \mathrm{a}, 4 \mathrm{~b})$ indicate to local $\mathrm{C}_{2 \mathrm{v}}$ symmetry $^{20}$ (shown in Figure 1). The $v(\mathrm{CO})$ modes in $(1 \mathrm{a}-4 \mathrm{a})$ and $(1 \mathrm{~b}-4 \mathrm{~b})$ move also to lower wave numbers when compared with the starting carbonyl complexes. ${ }^{13,14}$

In the ${ }^{1} \mathrm{H}$ NMR spectra of $(1 \mathrm{a}-4 \mathrm{a})$ and $(1 \mathrm{~b}-4 \mathrm{~b})$, the signal for the NH hydrogen of free ligands remain unchanged. No shift of hydroxyl protons with complex formation shows that $\mathrm{OH}$ group does not coordinate to metal atoms. The signals of the $\mathrm{HC}=\mathrm{N}$ and $\mathrm{CH}_{3}=\mathrm{N}$ protons show a small upfield shift, which may be related to changing $\pi$-electron density in the $\mathrm{C}=\mathrm{N}$ bond complex formation in $(1 \mathrm{a}-4 \mathrm{a})$ and $(1 \mathrm{~b}-4 \mathrm{~b})$. According to these data, $5 \mathrm{msalmsh}$ and $5 \mathrm{mafmsh}$ behave as monodentate ligands via the imine $\mathrm{N}$ donor atom in ( $1 \mathrm{a}-4 \mathrm{a})$ and $(1 \mathrm{~b}-4 \mathrm{~b})$.

The mass spectra show fragmentation via successive loss of CO groups and fragmentation of the organic ligands.

\section{Conclusions}

Eight new metal carbonyl complexes, $\left[\mathrm{M}(\mathrm{CO})_{5}(5 \mathrm{~m}\right.$ salmsh)] [M=Cr;(1a), Mo;(2a); W(3a)], $\left[\operatorname{Re}(\mathrm{CO})_{4} \operatorname{Br}(5 \mathrm{~m}\right.$ salmsh)] (4a), [M(CO) $\left.)_{5}(5 \mathrm{mafmsh})\right][\mathrm{M}=\mathrm{Cr} ;(1 \mathrm{~b}), \mathrm{Mo} ;(2 \mathrm{~b})$; $\mathrm{W}(3 \mathrm{~b})],\left[\operatorname{Re}(\mathrm{CO}){ }_{4} \operatorname{Br}(5 \mathrm{mafmsh})\right](4 \mathrm{~b})$, have been characterized using elemental analysis, $1 \mathrm{H}$ NMR, LC-MS and IR spectra. $5 \mathrm{msalmsh}$ and $5 \mathrm{mafmsh}$ behave as monodentate ligands via the $\mathrm{N}$ imine donor atom in (1a-4a) and (1b$4 \mathrm{~b})$. IR and NMR spectra of the compounds show that the $5 \mathrm{msalmsh}$ and $5 \mathrm{mafmsh}$ ligands coordinate to the metal atom for compounds $(1 \mathrm{a}-4 \mathrm{a})$ and $(1 \mathrm{~b}-4 \mathrm{~b})$ only via an imine $\mathrm{N}$ donor atom behaving as a monodentate neutral ligand. ${ }^{13}$

\section{Acknowledgments}

We thank BP (Turkey) for the provision of the photochemical apparatus and the ResearchFoundation of Ege University for funds and TUBITAK for allocation of time at the NMR, Mass Spectra and Elemental Analyses.

\section{References}

1. S. Rollas, N. Gulerman, H. Erdeniz, Farmaco, 2002, 57(2), 171-174. DOI:10.1016/S0014-827X(01)01192-2

2. Y. Ozama, N. H. Sugi, T. Nagasu, T. Owa, T. Watanabe, N. Koyanagi, H. Yoshino, K. Kitoh, K. Yoshimatsu, Eur. J. Cancer, 2001, 37, 2275-2282.

DOI:10.1016/S0959-8049(01)00275-1

3. U. A. K. Betz, R. Fischer, G. Kleymann, M. Hendrix, H. Rübsamen-Waigmann, Antimicrob. Agents Chemother., 2002, 46, 1766-1772. DOI:10.1128/AAC.46.6.1766-1772.2002

4. G. Bhattacharya, J. Herman, D. Delfin, M. M. Salem, T. Barszcz, M. Mollet, G. Riccio, R.Brun, K. A. Werbovetz, J. Med. Chem., 2004, 47, 1823-1832.

DOI:10.1021/jm0304461

5. L. M. Lima, E. G. Amarante, A. L. P. Miranda, C. A. M. Fraga, E. J. Barreiro, Pharm. Pharmacol. Commun., 1999, 5, 673-678. DOI:10.1211/146080899128734370

6. Ü. Ö. Özdemir, F. İlbiz, A. B. Gündüzalp et. al., Journal of Molecular Structure, 2015, 1100, 464-474.

DOI:10.1016/j.molstruc.2015.07.054

7. S. Özçelik, M. Dinçer, M. Şekerci, A. Balaban, Ü. Özdemir, Acta Crystallogr. Sect. E, 2004, 60(9), 1552-1553.

DOI:10.1107/S1600536804019932

8. L. L. Silva, K. N. Oliveria, R. J. Nunes, Arkivoc, 2006, 13, 124129. DOI:10.3998/ark.5550190.0007.d12

9. X. He, L. Tang, L. He, P. Xu, Hua Xi Yi Da Xue Bao, 1988, 19(3), 317-319. DOI:10.1039/b104037p

10. H. Lu, M. A. Schmidt, K. F. Jensen, Royal Society of Chemistry, 2001, 1, 22-28.

11. G. Orhan, O. S. Senturk, U. O. Ozmen, S. Sert, E. Subası, Journal of Coor. Chem., 2014, 67(19), 3216-3225.

DOI:10.1080/00958972.2014.963569

12. Marc Wrighton, Chemical Reviews, 1974, 74(4), 401-430. DOI:10.1021/cr60290a001

13. Ü. Ö. Özdemir, P. Güvenç, E. Şahin, F. Hamurcu, Inorganic Chimica Acta, 2009, 362, 2613-2618.

DOI:10.1016/j.ica.2008.11.029

14. M. J. Almond, F. Sarıkahya, O. S. Şentürk, Polyhedron, 1997, 16, 1101. DOI:10.1016/S0277-5387(96)00384-1

15. F. Sarıkahya, O. S. Şentürk, Synth. React. Inorg. Met.-Org. Chem., 2001, 31, 1843-1851.

16. F. A. Cotton, G. Wilkinson, G, Advanced Inorganic Chemistry, 1988, $5^{\text {th }}$ Ed., Wiley Interscience, NewYork, 1047. 
17. J. E. Kovacic, Spectrachim. Acta Part A: Molecular Spectroscopy, 1967, 23, 183-187. DOI:10.1016/0584-8539(67)80219-8

18. N. I. Dodoff, Ü. Özdemir, N. Karacan, M. Georgieva, S. M. Konstantinov, M. E. Stefanova, Z. Naturforsch, 1999, 54(12), 1553-1562. DOI:10.1515/znb-1999-1213
19. G. L. Miessler, D. A. Tarr, Inorganic Chemistry, 2000, $2^{\text {nd }}$ ed., Prentice Hall, New Jersey, 471.

20. U. Mazzi, A. Binmondo, N. Kotsev, D.A. Clemente, Journal of Organomet. Chem., 1977, 135(2), 177-182.

DOI:10.1016/S0022-328X(00)80858-X

\section{Povzetek}

Osem novih kompleksnih spojin $\left[\mathrm{M}(\mathrm{CO})_{5}(5 \mathrm{msalmsh})\right][\mathrm{M}=\mathrm{Cr} ;(1 \mathrm{a}), \mathrm{Mo} ;(2 \mathrm{a}) ; \mathrm{W}(3 \mathrm{a})],\left[\operatorname{Re}(\mathrm{CO})_{4} \mathrm{Br}(5 \mathrm{msalmsh})\right](4 \mathrm{a})$, $\left[\mathrm{M}(\mathrm{CO})_{5}(5 \mathrm{mafmsh})\right][\mathrm{M}=\mathrm{Cr} ;(1 \mathrm{~b}), \mathrm{Mo} ;(2 \mathrm{~b}) ; \mathrm{W}(3 \mathrm{~b})]$ in $\left[\operatorname{Re}(\mathrm{CO})_{4} \operatorname{Br}(5 \mathrm{mafmsh})\right](4 \mathrm{~b})$ smo sintetizirali s fotokemijsko reakcijo med kovinskimi karbonili, $\left[\mathrm{M}(\mathrm{CO})_{6}\right](\mathrm{M}=\mathrm{Cr}, \mathrm{Mo}, \mathrm{W}),\left[\operatorname{Re}(\mathrm{CO})_{5} \mathrm{Br}\right]$ in ligandoma (5msalmsh in $5 \mathrm{mafm}-$ sh). Spojine smo karakterizirali z elementno analizo, s tekočinsko kromatografijo sklopljeno z masno spektroskopijo (LC-MS), infrardečo spektroskopijo (FT-IR) in NMR spektroskopijo ( ${ }^{1} \mathrm{H}$ NMR). Rezultati spektroskopskih metod kažejo, da sta monodentatna liganda 5 msalmsh in 5 mafmsh na kovinski center v spojinah (1a)-(4a) in (1b)-(4b) koordinirana $\mathrm{z}$ atomom dušika, ki pripada imino skupini. 